\title{
Validation of the Monte Carlo simulator GATE for indium-111 imaging
}

\author{
K Assié ${ }^{1,2}$, I Gardin ${ }^{2,3}$, P Véra ${ }^{2,3}$ and I Buvat ${ }^{1}$ \\ ${ }^{1}$ UMR 678 INSERM / UPMC, CHU Pitié Salpêtrière, 91 boulevard de l'Hôpital, \\ 75634 Paris Cedex 13, France \\ ${ }^{2}$ Laboratoire Universitaire QUANT.I.F, Faculté de Médecine, 22 boulevard Gambetta, \\ 76183 Rouen Cedex, France \\ ${ }^{3}$ Département de Médecine Nucléaire, CHU et Centre Henri Becquerel, 1 rue d'Amiens, \\ 76000 Rouen, France \\ E-mail: buvat@imed.jussieu.fr
}

Received 20 November 2004, in final form 9 May 2005

Published 22 June 2005

Online at stacks.iop.org/PMB/50/3113

\begin{abstract}
Monte Carlo simulations are useful for optimizing and assessing single photon emission computed tomography (SPECT) protocols, especially when aiming at measuring quantitative parameters from SPECT images. Before Monte Carlo simulated data can be trusted, the simulation model must be validated. The purpose of this work was to validate the use of GATE, a new Monte Carlo simulation platform based on GEANT4, for modelling indium-111 SPECT data, the quantification of which is of foremost importance for dosimetric studies. To that end, acquisitions of ${ }^{111}$ In line sources in air and in water and of a cylindrical phantom were performed, together with the corresponding simulations. The simulation model included Monte Carlo modelling of the camera collimator and of a back-compartment accounting for photomultiplier tubes and associated electronics. Energy spectra, spatial resolution, sensitivity values, images and count profiles obtained for experimental and simulated data were compared. An excellent agreement was found between experimental and simulated energy spectra. For source-to-collimator distances varying from 0 to $20 \mathrm{~cm}$, simulated and experimental spatial resolution differed by less than $2 \%$ in air, while the simulated sensitivity values were within $4 \%$ of the experimental values. The simulation of the cylindrical phantom closely reproduced the experimental data. These results suggest that GATE enables accurate simulation of ${ }^{111}$ In SPECT acquisitions.
\end{abstract}

(Some figures in this article are in colour only in the electronic version) 


\section{Introduction}

Internal radiotherapy is a promising therapeutic approach (Erdi et al 1996). Calculation of the energy delivered to critical organs and tumours is essential to predict both organ toxicity and tumour response. The current practice (Siegel et al 1999) is to perform dosimetry by combining planar whole-body imaging, used to determine absolute activity in source organs, with MIRD S-factors derived from five anthropomorphic phantom models (Stabin 1996). A limit of this approach is the inaccuracy of activity measurements obtained from planar imaging. Furthermore, S-factors are derived from anthropomorphic phantoms and are not patient specific. They are tabulated for specific organs and not for tumours, which makes it difficult to use them for estimating dose in tumours. Several authors have underlined the need for truly patient-specific three-dimensional (3D) dosimetry (Sgouros et al 1990, Giap et al 1995, Bolch et al 1999, Gardin et al 2003). This might be achieved using accurate activity quantification of single photon emission tomography (SPECT) images and patient-specific S-factors.

In the context of internal radiotherapy, indium-111 $\left({ }^{111} \mathrm{In}\right.$, half life of $\left.67 \mathrm{~h}\right)$ has been used as a therapeutic agent in the treatment of primary and metastatic somatostatin receptorrich tumours with $\left[{ }^{111}\right.$ In-DTPA $\left.{ }^{0}\right]$ octreotide (Krenning et al 1996). Although it emits Auger electrons, ${ }^{111} \mathrm{In}$ is not optimal for therapy. The use of yttrium $90\left({ }^{90} \mathrm{Y}\right.$, half life of $\left.64 \mathrm{~h}\right)$ has been proposed instead (De Jong et al 1997). The drawback of ${ }^{90} \mathrm{Y}$ is that it cannot be used for imaging and dosimetry calculations as it is a pure beta emitter. ${ }^{90}$ Y-DOTATOC was thus proposed as a radiopharmaceutical for radiotherapy associated with ${ }^{111}$ In-DOTATOC for imaging in patients with octreotide receptor-positive lesions. More recently, ${ }^{90} \mathrm{Y}-\mathrm{Zevalin}{ }^{\circledR}$ was proposed for the treatment of non-Hodgkin's lymphoma (Wiseman et al 1999, Wiseman et al 2000). Preclinical studies demonstrated that ${ }^{111} \mathrm{In}-Z$ Zevalin ${ }^{\circledR}$ and ${ }^{90}$ Y-Zevalin ${ }^{\circledR}$ have similar biodistributions (Gansow et al 1991, Chinn et al 1999). Because ${ }^{111}$ In-Zevalin ${ }^{\circledR}$ can serve as a surrogate for ${ }^{90} \mathrm{Y}$, dosimetry studies using ${ }^{111} \mathrm{In}$-Zevalin ${ }^{\circledR}$ were used to estimate the radiation absorbed dose in ${ }^{90}$ Y-Zevalin ${ }^{\circledR}$ treatment. Accurate quantification of SPECT ${ }^{111} \mathrm{In}$ images is thus a prerequisite for dosimetry of radiopharmaceutical labelled with ${ }^{90} \mathrm{Y}$.

A relevant and powerful approach for studying quantitative accuracy in SPECT is the use of Monte Carlo simulations (Dewaraja et al 2000, Soret et al 2003). For instance, Monte Carlo simulations are extremely useful for studying and optimizing scatter correction methods, as simulations are the only mean to separate unscattered from scattered photons. Such simulations have actually been used for determining the accuracy with which tracer distribution can be estimated in ${ }^{111}$ In SPECT (Ljungberg et al 2003). However, to our knowledge, validation of Monte Carlo simulation codes in the context of ${ }^{111} \mathrm{In}$ SPECT, which is characterized by two emission energies (171 keV and $245 \mathrm{keV})$, has not been reported so far. The purpose of this study was thus to validate the Monte Carlo simulation code GATE (Staelens et al 2003, Assié et al 2004, Jan et al 2004, Lazaro et al 2004) for SPECT ${ }^{111}$ In imaging, as a first step towards designing optimized imaging protocols for reliable quantification in ${ }^{111}$ In SPECT. This validation study is based on the comparison between experimental and simulated data, in terms of energy spectra, spatial resolution, sensitivity and reconstructed SPECT images.

In section 2, we describe the acquisitions and corresponding simulations that have been performed, and the features used to assess the consistency between simulated and real data. Section 3 presents the values of different figures of merit for simulated and real data, while section 4 discusses the results and the reliability of the simulations. 
(a)

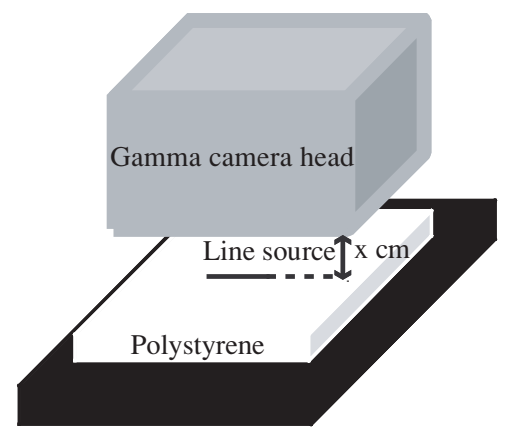

(b)

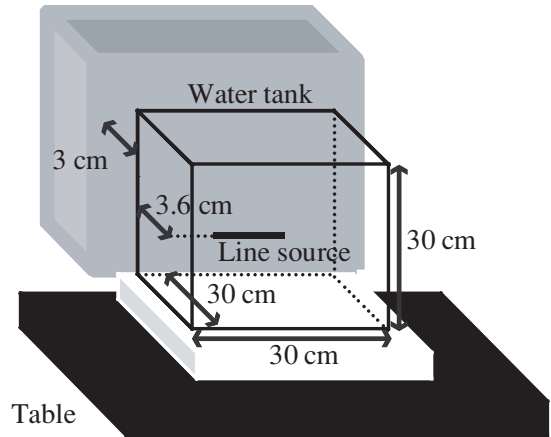

Figure 1. Set-up of acquisitions involving LINE1 and LINE2 (a) and LINE1w and LINE2w (b).

\section{Materials and methods}

\subsection{Experimental data}

2.1.1. Phantoms. Five phantoms were used. The phantom LINE1 consisted of a $6 \mathrm{~cm}$ long line source (inner diameter of $1.1 \mathrm{~mm}$ ) filled with $3.7 \mathrm{MBq}$ of ${ }^{111} \mathrm{In}$ and placed on a $4 \mathrm{~cm} \times$ $40 \mathrm{~cm} \times 40 \mathrm{~cm}$ polystyrene block (density of $100 \mathrm{mg} \mathrm{cm}^{-3}$ ) (figure 1(a)). The phantom LINE1w consisted of the same line source placed in a $30 \mathrm{~cm} \times 30 \mathrm{~cm} \times 30 \mathrm{~cm}$ water tank at $3.6 \mathrm{~cm}$ from the surface of the water. The water tank was placed on the polystyrene block (figure 1(b)). The phantom LINE2 consisted of a $5 \mathrm{~cm}$ long line source (inner diameter of $1.1 \mathrm{~mm}$ ) filled with $1.3 \mathrm{MBq}$ of ${ }^{111} \mathrm{In}$ and placed on a $4 \mathrm{~cm} \times 40 \mathrm{~cm} \times 40 \mathrm{~cm}$ polystyrene block (density of $100 \mathrm{mg} \mathrm{cm}^{-3}$ ) (figure 1(a)). The phantom LINE2w consisted of the same line source placed in a $30 \mathrm{~cm} \times 30 \mathrm{~cm} \times 30 \mathrm{~cm}$ water tank at $3.6 \mathrm{~cm}$ from the surface of the water. The water tank was placed on the polystyrene block (figure 1(b)).

The phantom CYL consisted of a cylindrical phantom (Deluxe Jaszczack Phantom ${ }^{\mathrm{TM}}$, $18.6 \mathrm{~cm}$ high and $22.25 \mathrm{~cm}$ in diameter) including five spheres (inner diameters of 33.5, 28, $16,13.5,10.5 \mathrm{~mm}$ respectively) with activity set to $129.5 \mathrm{kBq} \mathrm{ml}^{-1}$ (figure 2). A cold bony insert with a truncated cone shape $(45 \mathrm{~mm}$ long, 53.5 and $37 \mathrm{~mm}$ in diameter at the two ends, density of $1.93 \mathrm{~g} \mathrm{~cm}^{-3}$ ) was introduced at the centre of the phantom. Background activity was $15.8 \mathrm{kBq} \mathrm{ml}^{-1}$. The sphere-to-background activity concentration ratio was 8.2.

2.1.2. Acquisitions and reconstructions. A DST-XLi camera (General Electric Medical Systems) (3"8 NaI(Tl) crystal, FOV $=540 \mathrm{~mm} \times 400 \mathrm{~mm})$ equipped with two medium energy high resolution (MEHR) collimators was used to perform planar and SPECT acquisitions. All measurements involving different sources were performed sequentially.

Energy spectra measurements. LINE1 and LINE1w were placed at $10 \mathrm{~cm}$ and $6.6 \mathrm{~cm}$ from the collimator respectively (figure 1) (distance between the surface of the water and the collimator was $3 \mathrm{~cm}$ for LINE1w). For both LINE1 and LINE1w, planar data were acquired in 32 energy windows from 48 to $282 \mathrm{keV}$ ( 12 windows of $8 \mathrm{keV}$ from 48 to $144 \mathrm{keV}$, seven windows of $6 \mathrm{keV}$ from 144 to $186 \mathrm{keV}$, three windows of $10 \mathrm{keV}$ from 186 to $216 \mathrm{keV}$, one window of $8 \mathrm{keV}$ from 216 to $224 \mathrm{keV}$, seven windows of $6 \mathrm{keV}$ from 224 to $266 \mathrm{keV}$, two windows of $8 \mathrm{keV}$ from 266 to $282 \mathrm{keV}$ ) leading to 844 kcounts and 1.13 Mcounts, for LINE1 and LINE1w respectively. 


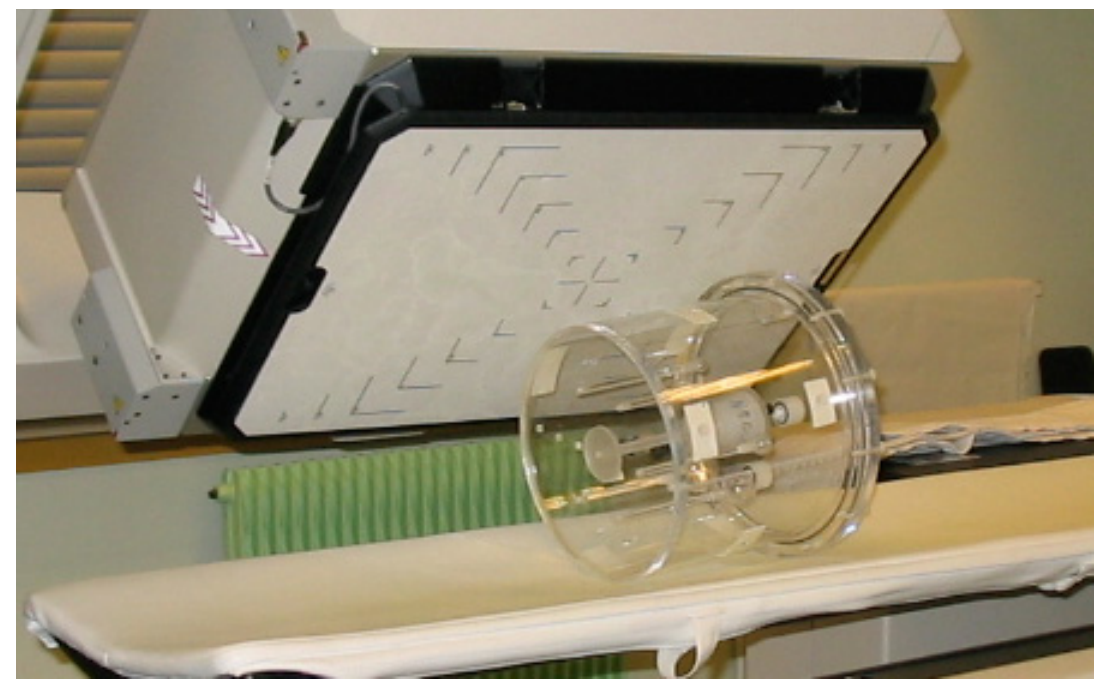

Figure 2. Experimental set-up involving the cylindrical phantom including spheres and a bony insert.

The CYL phantom was positioned centred in the transaxial field of view, with its axis parallel to the rotation axis of the gamma camera (figure 2). 128 projections (20 s per projection, matrix size $128 \times 128$, pixel size $=4.52 \mathrm{~mm} \times 4.52 \mathrm{~mm}$ ) were acquired along a circular orbit (radius of rotation $=25.2 \mathrm{~cm}$ ) over $360^{\circ}$, using 32 energy windows from 93 to $290 \mathrm{keV}$ ( 17 windows of $6 \mathrm{keV}$ from 93 to $198 \mathrm{keV}$, one window of $8 \mathrm{keV}$ from 198 to $206 \mathrm{keV}, 14$ windows of $6 \mathrm{keV}$ from 206 to $290 \mathrm{keV}$ ). About 40.8 Mcounts were acquired.

Spatial resolution and sensitivity measurements. Planar acquisitions of LINE2 and LINE2w were performed in the two $20 \%$ energy windows centred on 171 and $245 \mathrm{keV}$ respectively $($ matrix size $512 \times 512$, pixel size $=1.13 \mathrm{~mm} \times 1.13 \mathrm{~mm})$. The experimental set up was similar to that used for energy spectrum measurement, except that for LINE2 the phantom was placed at $0,5,10,15$ and $20 \mathrm{~cm}$ from the collimator, to measure the variation of the full width at half maximum (FWHM) and of the full width at tenth maximum (FWTM) with the source-to-collimator distance. For LINE2w, the distance between the surface of the water tank and the collimator was $3 \mathrm{~cm}$ (line source-to-collimator distance of $6.6 \mathrm{~cm}$ ). For all these acquisitions, about 300 and 250 kcounts were acquired in the 171 and $245 \mathrm{keV}$ energy windows respectively. Planar acquisitions of LINE2 were also used for sensitivity measurements.

Background measurement and correction. A 3 min planar acquisition was performed for background activity measurement, without any activity in the field of view of the detector. All planar data were systematically corrected from background activity by subtracting the background image after scaling to account for the difference in acquisition duration. Similarly, a SPECT acquisition of background activity was performed, with the same acquisition parameters as those of the CYL SPECT acquisition. All SPECT projections were systematically corrected from background activity by subtracting the projections of this background acquisition. 


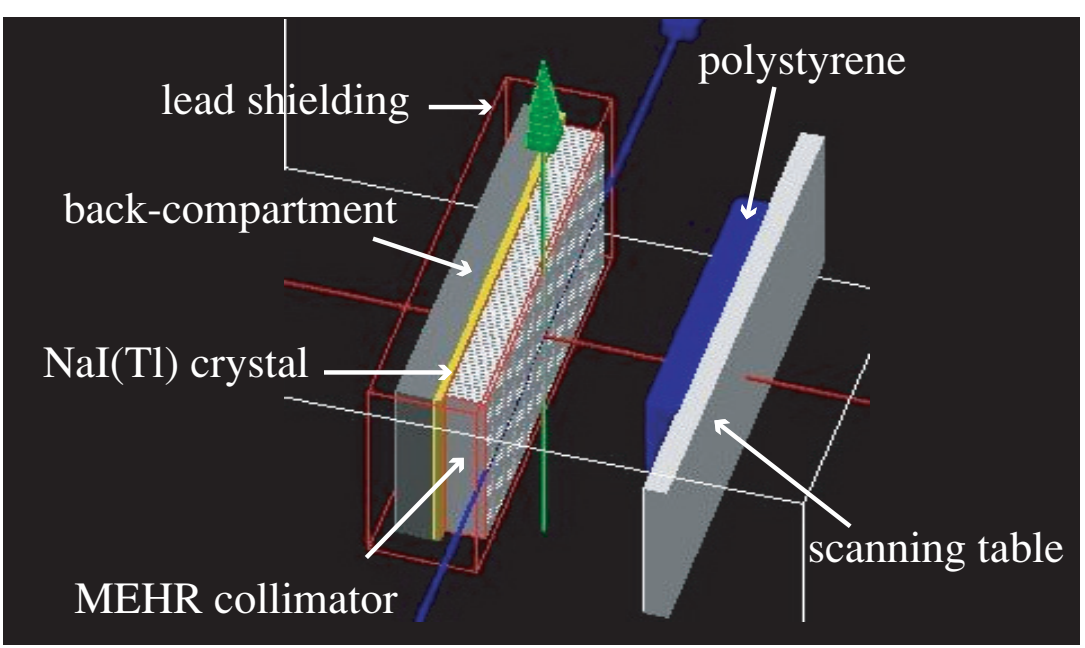

Figure 3. Gamma camera DST-Xli as modelled by GATE.

\subsection{Simulations}

The Monte Carlo simulation code GATE (Santin et al 2003, Assié et al 2004, Jan et al 2004, http://www.opengatecollaboration.org) was used. GATE allows for an accurate Monte Carlo modelling of photon transport in the phantom and in the camera collimator, crystal, head shielding and scanning table. It also makes it possible to model a so-called back-compartment to account for the photomultipliers and electronics located behind the crystal.

2.2.1. Camera modelling. The DST-XLi gamma camera was modelled as a combination of (figure 3):

- two MEHR lead collimators (55 mm thick, $3 \mathrm{~mm}$ flat-to-flat distance of the hexagonal holes and $0.6 \mathrm{~mm}$ septal thickness),

- a $3^{\prime \prime} 8$ thick $\mathrm{NaI}(\mathrm{Tl})$ crystal,

- a back-compartment, modelled as a $5 \mathrm{~cm}$ Perspex layer (density $2.5 \mathrm{~g} \mathrm{~cm}^{-3}$ ), accounting for the multipliers and associated electronics card,

- a head shielding in lead, $40 \mathrm{~mm}$ thick around the head and $30 \mathrm{~mm}$ thick on the rear,

- a scanning table, modelled as a $3.065 \mathrm{~cm}$ thick parallelepiped of polystyrene with a $100 \mathrm{mg} \mathrm{cm}^{-3}$ density.

To illustrate the importance of modelling the back-compartment, this model (GATE model I) will be compared to the same model without including the back-compartment (GATE model II).

Photons were tracked in all components of the gamma camera heads (collimator, crystal, back-compartment and shielding). For each photon reaching the crystal, all interactions of the photon in the crystal were recorded. The location of the detected event was then given by the centroid of all interaction points in the crystal weighted by the ratio of the deposited energy to the total energy deposited in the crystal. The energy associated with the detected event was equal to the sum of all deposited energies.

The spatial resolution blur (FWHM electronics $)$ introduced by the electronics processing was modelled by the convolution of the output data using a $1.6 \mathrm{~mm}$ FWHM bidimensional Gaussian function. This value was determined by comparing the known intrinsic spatial resolution of 
the camera $\left(\mathrm{FWHM}_{\text {intrinsic }}=3.4 \mathrm{~mm}\right)$ with the spatial resolution blur introduced by the crystal only $\left(\mathrm{FWHM}_{\text {crystal }}=3.0 \mathrm{~mm}\right)$, the latter being estimated by the simulation:

$$
\mathrm{FWHM}_{\text {electronics }}=\sqrt{\mathrm{FWHM}_{\text {intrinsic }}^{2}-\mathrm{FWHM}_{\text {crystal }}^{2}} .
$$

The energy resolution $\mathrm{FWHM}_{\mathrm{E}}$ of the camera was assumed to depend on energy $E$ in the following way:

$$
\mathrm{FWHM}_{\mathrm{E}}=\mathrm{FWHM}_{0} \sqrt{E_{0}} / \sqrt{E}
$$

with $\mathrm{FWHM}_{0}=10 \%$ at energy $E_{0}=245 \mathrm{keV}$. This value was determined by analysing the energy spectrum of a point source in air.

2.2.2. Acquisition modelling. The line sources and the cylindrical phantom were analytically described to serve as an input for GATE. To model the two emission energies of ${ }^{111}$ In (171 and $245 \mathrm{keV}$ ), two independent simulations were performed and appropriately weighted to account for the respective importance of the 171 and 245 emission rays.

The phantom experiments were simulated, using the very same geometrical configurations as for the real experiments, including the polystyrene blocks for the line source experiments.

For both LINE1 and LINE1w, 500 million emission events were simulated at each emission energy. The total number of detected events was about 121 kcounts for LINE1 and about 225 kcounts for LINE1w between 48 and $282 \mathrm{keV}$.

For both LINE2 and LINE2w, 800 million emission events were simulated at each emission energy. The total number of events detected in the two $20 \%$ energy windows centred on 171 and $245 \mathrm{keV}$ was about 315 kcounts for LINE2 and about 101 kcounts for LINE2w.

For CYL, 30 billion emission events were simulated. About 197 kcounts were detected in the two $20 \%$ energy windows centred on 171 and $245 \mathrm{keV}$.

For comparison purpose, two configurations (LINE1 and LINE2 at $10 \mathrm{~cm}$ from the collimator) were also modelled using the SimSET Monte Carlo simulator (Harrisson et al 1993). Unlike GATE, SimSET does not allow for Monte Carlo simulation of the photon transport within the collimator and only includes an analytical model of the collimator response. The SimSET model is the same as GATE model I but with an analytical modelling of MEHR collimators instead of a Monte Carlo modelling.

\subsection{Assessment of the simulation accuracy}

To assess the consistency between experimental and simulated data, three features were considered:

- Energy spectra. The energy spectra of events recorded in the whole field of view for LINE1, LINE1w and CYL were obtained and compared to those derived from the corresponding simulations.

- Spatial resolution. The FWHM and FWTM of line spread functions were estimated for the different source-to-collimator distances for LINE2 and at a $6.6 \mathrm{~cm}$ source-to-collimator distance for LINE2w, by drawing profiles $(22.6 \mathrm{~cm}$ thick) through the line source seen on the images.

- Sensitivity. The sensitivity defined as the number of detected events divided by the number of emitted or simulated events was calculated for the LINE2 configuration. Standard deviations of the simulated data were obtained by repeating each simulation five times and those of the experimental data were obtained by considering a $5 \%$ error in the dose calibrator. 
Number of detected counts (AU)

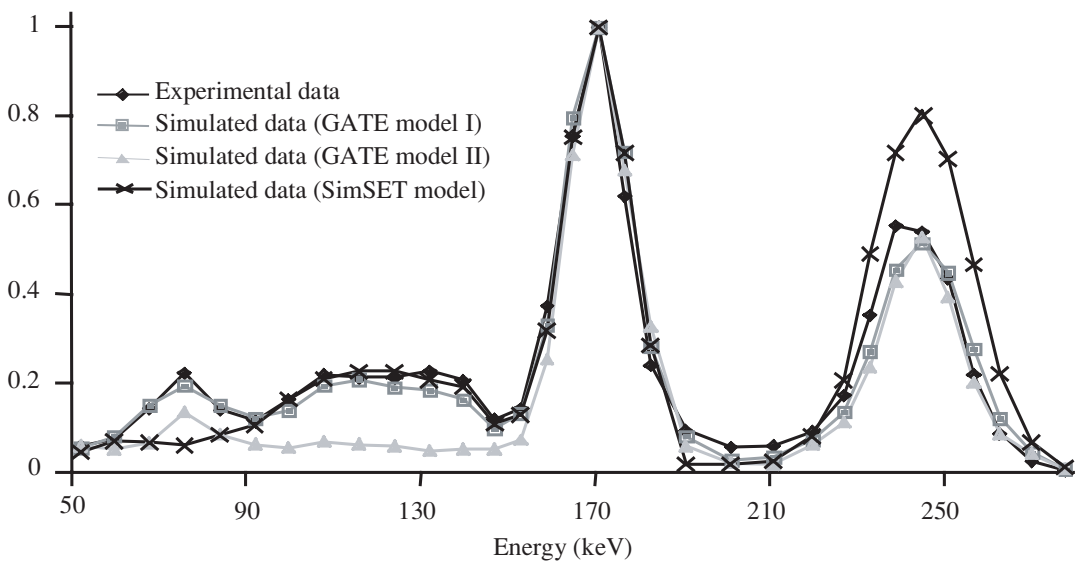

Figure 4. Acquired and simulated spectra of LINE1 using SimSET, GATE model I and GATE model II simulation models.

Using the CYL phantom data, simulated and acquired projections corresponding to the two $20 \%$ energy windows centred on 171 and $245 \mathrm{keV}$ were obtained by summing the appropriate energy windows among the 32 that were recorded. The projections corresponding to the two photopeaks were then summed and reconstructed using OSEM (four subsets, six iterations). The reconstructed voxel size was $4.52 \mathrm{~mm} \times 4.52 \mathrm{~mm} \times 4.52 \mathrm{~mm}$. The agreement between experimental and simulated data was semi-quantitatively assessed on a reconstructed slice passing through the centre of the spheres. An $18 \mathrm{~mm}$ thick profile through the largest sphere was drawn both on experimental and simulated data.

\section{Results}

All results presented hereafter pertain to GATE model I simulations, unless specified otherwise.

\subsection{Energy spectra}

Figure 4 shows the experimental and simulated energy spectra obtained for LINE1 normalized to the same maximum, for the different simulation models (SimSET, GATE model I and GATE model II). GATE model I appears to be the most appropriate to accurately reproduce the experimental data.

Figure 5 shows the experimental and simulated spectra normalized to the same maximum, obtained for LINE1w and CYL, when using GATE model I. This figure confirms that GATE model I accurately reproduces experimental data in various configurations.

Figure 6 shows the unscattered and scattered photon spectra of the detected events for LINE1 and LINE1w. The scatter events are sorted as a function of the compartment in which the last scattering occurred. This figure shows the relatively high contribution of events that last scattered in the back-compartment of the camera. Table 1 gives the percentages of unscattered and scattered photons, as a function of the medium in which the last scattering occurred, for LINE1 and LINE1w, confirming the need for modelling the back-compartment to accurately model scatter. 

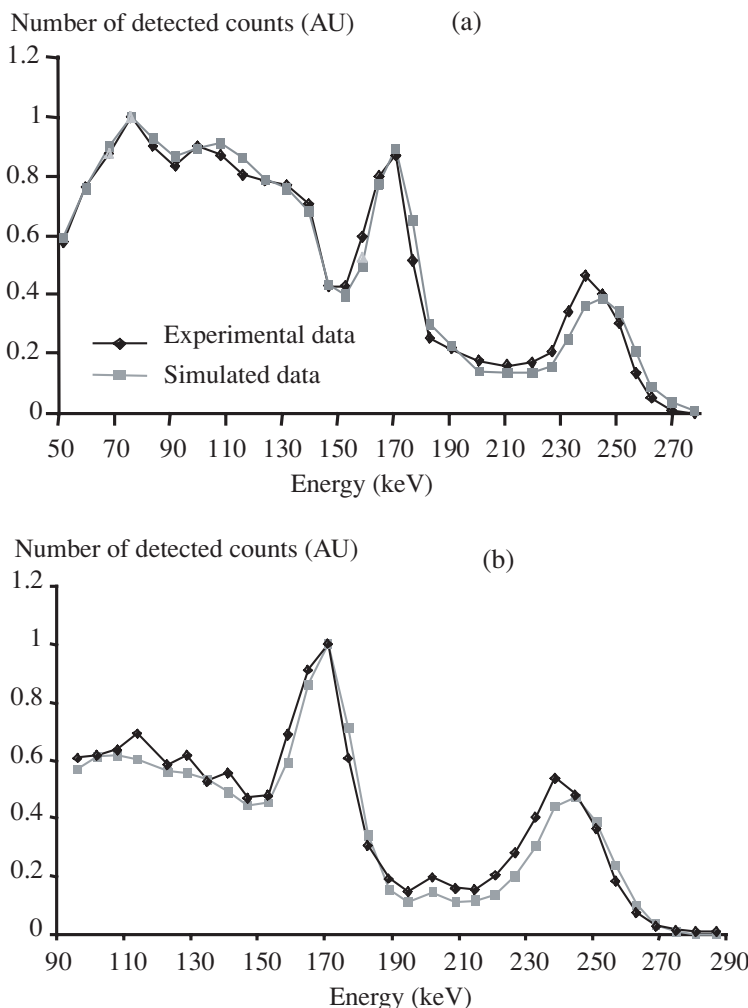

Figure 5. Acquired and simulated spectra of LINE1w (a) and CYL (b) when using GATE model I.

Table 1. Percentage of unscattered and scattered photons, as a function of the medium in which the last scattering occurred for LINE1 and LINE1w.

\begin{tabular}{llllllll}
\hline & & \multicolumn{5}{c}{ Scatter } \\
\cline { 3 - 7 } & Unscattered & Shielding & Collimator & Back-compartment & Scanning table & Polystyrene block & Water tank \\
\hline LINE1 & 76.5 & 0.3 & 2.6 & 16.9 & 1.6 & 2.1 & \\
LINE1w & 25.8 & 0.1 & 0.9 & 7.1 & 0.1 & 0.2 & 65.8 \\
\hline
\end{tabular}

\subsection{Spatial resolution}

The FWHM and FWTM of the line spread functions for the LINE2 configurations are given in table 2. The LINE2 configuration at $10 \mathrm{~cm}$ from the collimator was also simulated using SimSET for comparison purpose. The simulated FWHM with SimSET was $7.9 \mathrm{~mm}$, corresponding to a $16.8 \%$ difference with respect to the experimental value.

For the LINE2w configuration (line-source-collimator distance $=6.6 \mathrm{~cm}$ ), the experimental and simulated FWHM were 9.6 and $8.3 \mathrm{~mm}$ respectively, corresponding to a $13.5 \%$ difference. The experimental and simulated FWTM were $17 \mathrm{~mm}$ and $15.1 \mathrm{~mm}$ respectively, corresponding to a $11.2 \%$ difference. 
Number of detected counts (AU)

(a)

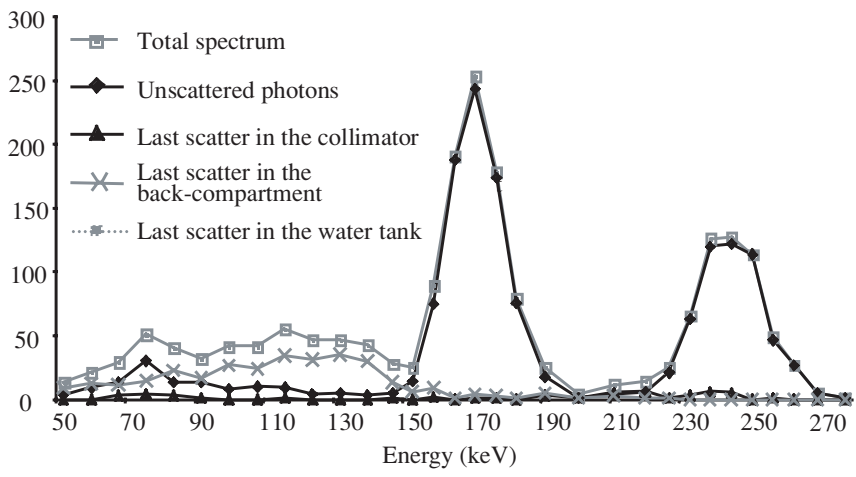

Number of detected counts (AU)

(b)

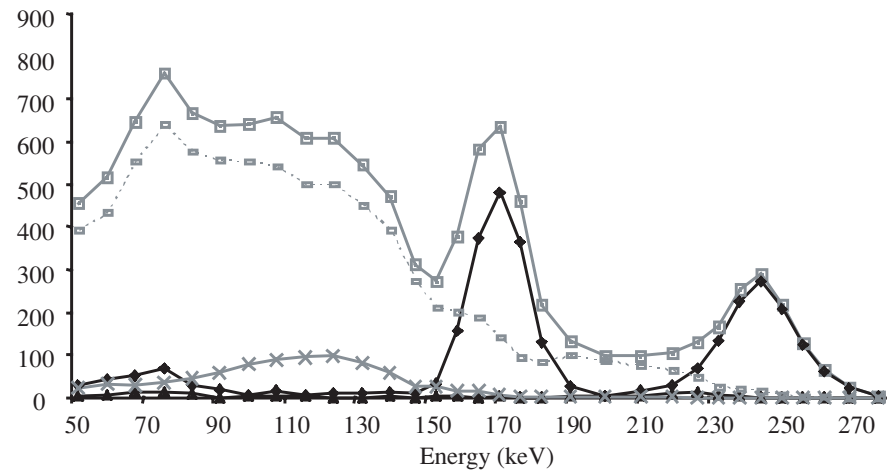

Figure 6. Simulated spectra of unscattered and scattered photons: LINE1 configuration (a) and LINE1w configuration (b). Scattered events sorted as a function of the medium in which the last scattering occurred (i.e. collimator, back-compartment, water tank) are shown.

Table 2. FWHM and FWTM in mm for different ${ }^{111}$ In line source-to-collimator distances obtained from simulations and real experiments for LINE2. Per cent differences between simulations and experimental data (with respect to the experimental value) are given in parentheses.

\begin{tabular}{llllll}
\hline & \multicolumn{4}{c}{ Source-to-collimator distance (mm) } \\
\cline { 2 - 5 } & 0 & 50 & 100 & 150 & 200 \\
\hline FWHM & & & & & \\
$\quad$ Simulations & $5.7(-1.7 \%)$ & $7(0 \%)$ & $9.6(1.1 \%)$ & $11.5(0 \%)$ & $14.4(1.4 \%)$ \\
$\quad$ Experiments & 5.8 & 7 & 9.5 & 11.5 & 14.2 \\
FWTM & & & & $19.5(-3 \%)$ & $24.4(-1.6 \%)$ \\
$\quad$ Simulations & $9.5(20.3 \%)$ & $11.8(-7.8 \%)$ & $16(-4.8 \%)$ & 20.1 & 24.8 \\
$\quad$ Experiments & 7.9 & 12.8 & 16.8 & 2.8 \\
\hline
\end{tabular}

\subsection{Sensitivity}

Figure 7 shows the simulated and measured sensitivity values in air and associated standard deviations for different source-to-collimator distances (LIGNE2 configurations). 


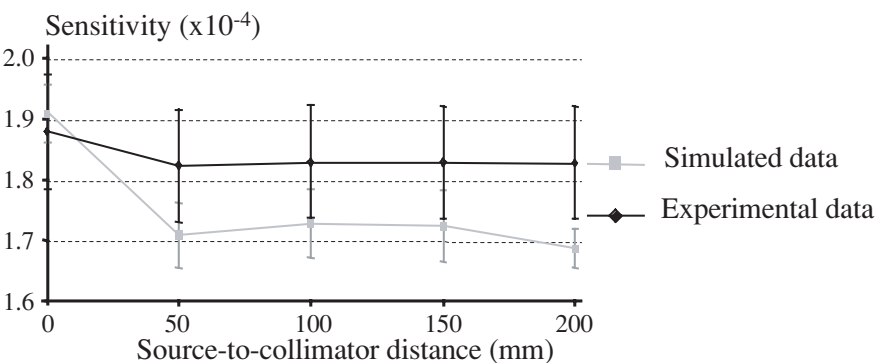

Figure 7. Sensitivity values for different ${ }^{111}$ In line source-to-collimator distances obtained from simulations and real experiments in air.

(a)

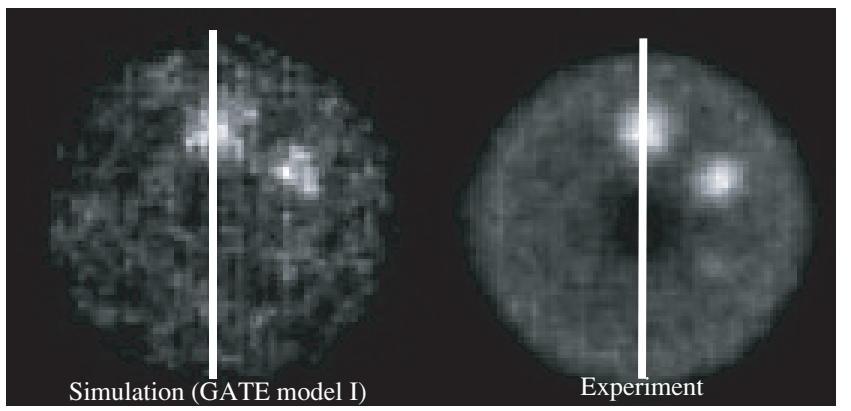

number of detected counts

1000

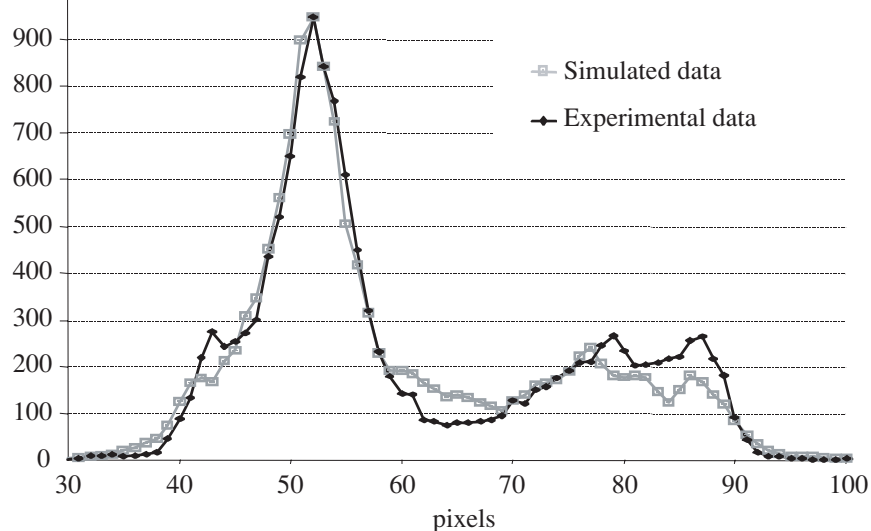

Figure 8. Slices through the spheres of the CYL phantom (a) and profiles (b) taken at the location shown on the images.

\subsection{Cylindrical phantom}

Figure 8 shows a reconstructed slice through the spheres of the CYL phantom and the profiles drawn through the largest sphere for the experimental and simulated data. The two profiles were normalized to the same maximum. 


\subsection{Computation time}

Total computation time of the GATE simulations was about $64 \mathrm{~h}$ CPU for LINE1, $111 \mathrm{~h}$ CPU for LINE2 and $600 \mathrm{~h} \mathrm{CPU}$ for CYL on a Pentium 4-2 GHz computer (cache $512 \mathrm{~KB}$ ).

\section{Discussion}

GATE is a recent simulation toolkit enabling the modelling of a large variety of configurations in both SPECT and PET (Assié et al 2004, Jan et al 2004). Of interest, the photon transport through the collimator can be modelled using Monte Carlo techniques, which is of foremost importance for realistic simulations of SPECT acquisitions involving high-energy radioisotopes. For this reason, we chose to consider GATE for modelling ${ }^{111}$ In SPECT acquisitions.

Because ${ }^{111}$ In emits gamma photons at both 171 and $245 \mathrm{keV}$ that can interact with various camera components, a realistic model of the gamma camera heads had to be designed. In particular, as some photons pass through the collimator and crystal without interacting, the physical interactions that might occur behind the crystal have to be modelled. Precise modelling of the photomultipliers and associate packaging is difficult, because of the various materials the photomultipliers are made of. We therefore designed a so-called 'backcompartment', the function of which was to act as a compartment in which interactions similar to those occurring in the photomultipliers and associated electronics card would occur. The parameters of the back-compartment were determined empirically so that the simulated energy spectrum matched the measured energy spectrum in the specific configuration of the LINE1 experiment: a parallelepiped $(5 \mathrm{~cm} \times 40.5 \mathrm{~cm} \times 56.3 \mathrm{~cm})$ filled with Perspex (density $2.5 \mathrm{~g} \mathrm{~cm}^{-3}$ ) was found to be appropriate. It was then checked that the low-energy spectra were accurately reproduced in very different configurations (see LINE1w and CYL figure 5), suggesting that the back-compartment model had a similar behaviour (with respect to the physical interactions it creates) as the actual camera components located behind the crystal. Figure 6 and table 1 suggest that the back-compartment is indeed responsible for most of the scatter interactions occurring in the camera head. The modelling of the other components of the gamma camera was rather straightforward, given the gamma camera description.

Comparison between simulated and real data shows that the GATE modelling accurately reproduced the experimental energy spectra (figures 4 and 5). However, there was a trend towards an underestimation of detected counts from $200 \mathrm{keV}$ and a slight shift between the experimental and simulated $245 \mathrm{keV}$ photopeaks. This was actually due to the distorted response of the gamma camera over the large energy range that was investigated. Indeed, looking at the location of the photopeak of ${ }^{99} \mathrm{~m} \mathrm{Tc}(140 \mathrm{keV}),{ }^{111} \mathrm{In}(171$ and $245 \mathrm{keV})$ and ${ }^{131} \mathrm{I}(364 \mathrm{keV})$ by performing point source acquisitions in air, we found that the energy at which these photopeaks were detected were 139, 170, 242 and $358 \mathrm{keV}$ respectively. The corresponding per cent differences between real and detected photopeak energies were $0.7 \%$, $0.6 \%, 1.2 \%$ and $1.6 \%$. The energy response of the camera was therefore not perfectly proportional to the energy deposited in the crystal, while the simulated energy response was. This distortion of the energy response of the camera could actually be modelled using the 'digitizer' in GATE (Jan et al 2004) if needed. The spectra presented in figures 4 and 5 show a non-negligible contribution of the lead x-rays at around $77 \mathrm{keV}$, suggesting that these rays have to be simulated to accurately reproduce experimental data below $100 \mathrm{keV}$ if such an energy range is of interest.

Spatial resolutions expressed as FWHM in air were predicted within 2\% (table 2). As expected, the sensitivity did not depend on the source-to-collimator distance in the 
experimental data and in the simulated data. The average per cent difference between simulated and experimental sensitivity values was $4.7 \% \pm 3.3 \%$, for source-to-collimator distance between 0 and $20 \mathrm{~cm}$. GATE was thus able to accurately predict the spatial resolution and the sensitivity of the imaging device.

Despite the low statistics of the simulated images of the cylindrical phantom (about 100 times less counts than in the real data), which explains the poor quality of the reconstructed image, a reasonable agreement was found between experimental and simulated images (figure 8), confirming that data corresponding to more complicated objects than line sources can be accurately simulated.

A relevant question is whether similar accuracy in modelling can be obtained with other Monte Carlo simulators. To address this question, we tried to determine which components of the model were essential to properly reproduce the experimental data. By considering the SimSET model that mostly differs from GATE model I by the analytical modelling of the collimator response, we demonstrated that such analytical modelling was insufficient to closely reproduce the experimental data. Monte Carlo transport of the photons through the collimator was essential to simulate realistic data. Similarly, comparing GATE model I with GATE model II allowed us to demonstrate the crucial role of the back-compartment modelling, without which large differences between simulated and experimental data were observed (figure 4). Other Monte Carlo codes allowing for Monte Carlo modelling of (1) the photon transport in the collimator, (2) the lead x-rays emitted by the collimator and (3) an ad hoc back-compartment to account for the detector components located behind the crystal, might also be appropriate to accurately simulate ${ }^{111}$ In data.

The major drawback of GATE is the computation time. This drawback really becomes an issue when using complicated voxelized geometries. A solution to speed up simulations could be the use of variance reduction techniques (Haynor 1998), of computer clusters or of 'gridification' (Breton et al 2003). These different solutions are currently under study in the OpenGATE collaboration (http://www.opengatecollaboration.org). These developments will probably determine the practical use of GATE for simulating ${ }^{111}$ In data for quantification studies in the context of dosimetry.

\section{Conclusion}

GATE enables accurate simulation of ${ }^{111}$ In SPECT acquisitions in terms of energy spectra, spatial resolution, sensitivity and modelling of realistic source distributions, thanks to appropriate Monte Carlo modelling of photon transport through the collimator and within a back-compartment located behind the crystal. By properly tuning the model of the imaging system, GATE can therefore be confidently used for the design and assessment of acquisition and processing protocols in ${ }^{111}$ In SPECT.

\section{Acknowledgment}

This work was supported by the French Ligue Nationale Contre le Cancer and by the GDR Stic-Santé (France).

\section{References}

Assié K et al 2004 Monte Carlo simulation in PET and SPECT instrumentation using GATE Nucl. Instrum. Methods. A $\mathbf{5 2 7} 180-9$

Bolch W E et al 1999 MIRD Pamplet no. 17: the dosimetry of nonuniform activity distributions-radionuclide S values at the voxel level J. Nucl. Med. $4011 \mathrm{~S}-36 \mathrm{~S}$ 
Breton V, Medina R and Montagnat J 2003 DataGrid, prototype of a biomedical grid Meth. Inf. Med. 42 143-7

Chinn P C, Leonard J E, Rosenberg J, Hanna N and Anderson D R 1999 Preclinical evaluation of 90Y-labeled anti-CD20 monoclonal antibody for treatment of non-Hodgkin's lymphoma Int. J. Oncol. 15 1017-25

De Jong M, Bakker W H, Krenning E P, Breeman W A, van der Pluijm M E and Bernard B F 1997 Yttrium-90 and indium-111 labelling, receptor binding and biodistribution of [DOTA0,D-Phe1,Tyr3]-octreotide, a promising somatostatin analogue for radionuclide therapy Eur. J. Nucl. Med. 24 368-71

Dewaraja Y K, Ljungberg M and Koral K F 2000 Accuracy of 131I tumor quantification in radioimmunotherapy using SPECT imaging with an ultra-high-energy collimator: Monte Carlo study J. Nucl. Med. 41 1760-7

Erdi A K, Erdi Y E, Yorke E D and Wessels B W 1996 Treatment planning for radio-immunotherapy Phys. Med. Biol. 41 2009-26

Gansow O A 1991 Newer approaches to the radiolabeling of monoclonal antibodies by use of metal chelates Int. J. Rad. Appl. Instrum. B 18 369-81

Gardin I, Bouchet L G, Assie K, Caron J, Lisbona A, Ferrer L, Bolch W E and Vera P 2003 Voxeldoes: a computer program for 3-D dose calculation in therapeutic nuclear medicine Cancer Biother. Radiopharm. 18 109-15

Giap H B, Macey D J and Podoloff D A 1995 Development of a SPECT-based three-dimensional treatment planning system for radioimmunotherapy $J$. Nucl. Med. 36 1885-94

GEANT4 Collaboration 2002 GEANT4: a simulation toolkit SLAC Report SLAC-PUB-9350

Haynor D R 1998 Variance reduction techniques Monte Carlo Calculations in Nuclear Medicine ed M Ljungberg, S E Strand and M A King (Bristol: Institute of Physics Publishing) pp 13-24

Harrisson R L, Vannoy S D, Haynor D R, Gillispie S B, Kaplan M S and Lewellen T K 1993 Preliminary experience with the photon history generator module of a public-domain simulation system for emission tomography Conf. Rec. IEEE Nucl. Sci. Symp. 2 1154-8

Jan S et al 2004 GATE: a simulation toolkit for PET and SPECT Phys. Med. Biol. 49 4543-61

Krenning E P, Kooij P P, Pauwels S, Breeman W A, Postema P T, De Herder W W, Valkema R and Kwekkeboom D J 1996 Somatostatin receptor: scintigraphy and radionuclide therapy Digestion 57 57-61

Lazaro D et al 2004 Validation of the GATE Monte Carlo simulation platform for modelling a CsI(Tl) scintillation camera dedicated to small-animal imaging Phys. Med. Biol. 49 271-85

Ljungberg M, Frey E, Sjogreen K, Liu X, Dewaraja Y and Strand S E 2003 3D absorbed dose calculations based on SPECT: evaluation for 111-In/90-Y therapy using Monte Carlo simulations Cancer Biother. Radiopharm. 18 99-107

Santin G, Strul D, Lazaro D, Breton V and Morel C 2003 GATE, a Geant4-based simulation platform for PET imaging integrating movement and time management IEEE Trans. Nucl. Sci. 50 1516-21

Sgouros G, Barest G, Thekkumthala J, Chui C, Mohan R, Bigler R E and Zanzonico P B 1990 Treatment planning for internal radionuclide therapy: three-dimentional dosimetry for nonuniformly distributed radionuclides $J$. Nucl. Med. 31 1884-91

Siegel J A et al 1999 MIRD pamphlet no. 16: techniques for quantitative radiopharmaceutical biodistribution data acquisition and analysis for use in human radiation dose estimates J. Nucl. Med. 40 37S-61S

Soret M, Koulibaly P M, Darcourt J, Hapdey S and Buvat I 2003 Quantitative accuracy of dopaminergic neurotransmission imaging with (123)I SPECT J. Nucl. Med. 44 1184-93

Stabin M 1996 MIRDOSE:personal computer software for internal dose assessment in nuclear medicine J. Nucl. Med. $37538-46$

Staelens S, Strul D, Santin G, Vandenberghe S, Koole M, D’Asseler Y, Lemahieu I and Van de Walle R 2003 Monte Carlo simulations of a scintillation camera using GATE: validation and application modelling Phys. Med. Biol. 48 3021-42

Wiseman G A et al 1999 Radioimmunotherapy of relapsed Non-Hodgkin's Lymphoma with ZEVALIN, a ${ }^{90}$ Y-labeled anti-CD20 monoclonal antibody Clin. Cancer Res. 5 3281s-6s

Wiseman G A et al 2000 Phase I/II 90Y-Zevalin (yttrium-90 ibritumomab tiuxetan, IDEC-Y2B8) radioimmunotherapy dosimetry results in relapsed or refractory non-Hodgkin's lymphoma Eur. J. Nucl. Med. 27 766-77 